\title{
Dosimetric model of human lung and associated computer program
}

\author{
D Nikezic $^{1 *}$ and $\mathrm{KN} \mathrm{Yu}{ }^{2}$ \\ ${ }^{1}$ University of Kragujevac, Faculty of Science, R. Domanovic 12, Kragujevac, Serbia \\ ${ }^{2}$ City University of Hong Kong, Department of Physics and Materials Science, Hong Kong, P. R. China \\ E-mail : nikezic@kg.ac.yu
}

\begin{abstract}
The dosimetric human respiratory tract model given in ICRP Publication 66 and ICRP Vol. 32 is briefly described in this paper. The associated home-written computer programs for calculations of radiation dose from radon and its short-lived progeny are presented, together with some representative results.
\end{abstract}

Keywords : Dosimetry, human lung, radon.

PACS Nos. : 87.53.Bn, 23.60.+e, 87.50.-a

\section{Introduction}

The main radioactive contaminant in the human environment is radon $\left.{ }^{222} \mathrm{Rn}\right)$. It has been estimated that about half of the total effective dose received by human beings from all sources of ionizing radiation is attributed to short-lived progeny of ${ }^{222} \mathrm{Rn}$ [1]. Natural levels of ${ }^{222} \mathrm{Rn}$ in open space are usually low as $10 \mathrm{Bqm}^{-3}$. However, in closed space, like indoor environments, ${ }^{222} \mathrm{Rn}$ is accumulated due to poor ventilation. High radon concentrations can represent significant radiation hazards. On decay of ${ }^{222} \mathrm{Rn}$, its short-lived progeny, ${ }^{218} \mathrm{Po}$ ( $\alpha$-particle emitter), ${ }^{214} \mathrm{~Pb}\left(\beta\right.$-particle emitter), ${ }^{214} \mathrm{Bi}$ ( $\beta$-particle emitter) and ${ }^{214} \mathrm{Po}$ ( $\alpha$-particle emitter) are formed. At the moment of creation in air, ${ }^{218} \mathrm{Po}$ is in the atomic stage, constituting the unattached (or free fraction of) radon progeny. The progeny tend to attach themselves to aerosols present in the air to form the attached fraction. Radon progeny will enter the human lungs during the inhalation and will be deposited there. The radiation emitted by the progeny damages the sensitive tissues in the lungs and may cause lung cancer. Some fractions of the radon progeny will also penetrate from the lungs into the blood and irradiate the whole human body. 
There are several special physical quantities and units used in radon science. The concentration of radon progeny is usually expressed through the quantity called potential alpha energy concentration (PAEC) defined as total energy of alpha particles emitted by the radon progeny in a unit volume of air. It is calculated from the formula :

$$
\mathrm{PAEC}=\sum_{i=1}^{4} C_{i} E_{i}
$$

where $C_{i}$ is the number of atoms of the $i$-th radon progeny in $1 \mathrm{~m}^{3}$ of air and $E_{i}$ is the corresponding potential alpha energy in Joule $(J)$. The SI unit for PAEC is $\mathrm{Jm}^{-3}$; while the traditional and very commonly used unit is working level, $\mathrm{WL},(1 \mathrm{WL}=2.08$ $\times 10^{-5} \mathrm{Jm}^{-3}$ ). The exposure to radon progeny $\boldsymbol{X}$ is the product of the average PAEC and the exposure time $t$ of the exposure :

$$
x=\mathrm{PAEC} \times t
$$

and expressed in $\mathrm{Jsm}^{-3}$. The traditional unit in use is called the working level month (WLM) and represents exposure to the radon progeny of $1 \mathrm{WL}$ during $170 \mathrm{~h}$.

The exposure of the human body to radiation from radon and its progeny results in an absorbed dose in some sensitive tissues in the lung. By weighting of absorbed dose with radiation weighting factors and tissue weighting factors, an effective dose, $E$, is obtained. Determination of the effective dose in the human lung for a given exposure condition is a very complicated and complex task. There are many parameters involved in the calculation of the effective dose in the human lung which will be discussed below. In addition to PAEC and exposure, some more quantities and terms are in use in this field as follows :

Intake is the activity that enters the body.

Uptake is a fraction of activity that enters the body that is absorbed in the blood and other body fluids and organs.

Dose coefficient is committed effective dose (in Siverts, Sv) to an organ resulting from intake of unit activity (1 Bq) (i.e., dose per unit intake DPUI).

Dose conversion coefficient is similar to dose coefficient but is given in $\mathrm{mSv}$ per WLM.

Biokinetic model is a set of mathematical equations that describes behavior of radionuclides in human body.

Dosimetric model calculates the energy absorbed in organs and tissues and hence the committed doses.

\section{Short history of dosimetric models for human respiratory tracts}

The lung is an important route for airborne radionuclides to enter the human body, for workers, as well as, for general population. This route is particularly important in the case of environmental and indoor radon and its short lived progeny. Inhaled radionuclides 
irradiate sensitive tissue of the lung, and can also be transferred to the blood and then irradiate other organs.

The first necessity for a dosimetric model appeared just after the 2nd World War for the purpose of protection of workers employed in uranium mining and processing. The first conference was held in Chalk River, Canada, in 1949 and the general lack of information was noticed. Tripartite Conference on Radiation protection was held from 1949 to 1953. Their consideration was summarized in ICRP publication 2 (ICRP 1960) [2] where the simple model of deposition, retention and clearance was presented and used as a basis for the limits for exposure, and dose calculations and prediction.

Later, ICRP formed a Task Group on Lung Dynamics to review the model, and significantly improved the model as published in 1966 (TLGD 66) [3]. This model was slightly modified in ICRP Publication 30 (ICRP 79) [4]. The main point was a new classification of chemical compound in $\mathrm{D}, \mathrm{W}, \mathrm{Y}$; class $\mathrm{D}$ means highly soluble particles cleared from the lung with half times less than 1 day; class $W$ cleared within 1 week and class $Y$ means very insoluble particles cleared between 6 months to 1 year. This model was the basis for dose calculations for inhaled radionuclides. This leads to recommendations of annual limits on intake of radionuclides.

The model motivated extensive investigation in this field. Some criticisms appeared, particularly on (i) the D,W Y classification scheme, (ii) that ICRP 30 was intended for protection of workers, and public protection was necessary, and (iii) that the model considered only adult Caucasian and it was not possible to apply it to other members of the population and other ethnic groups.

Extensive experimental and theoretical results were summarized in the new ICRP lung model published as ICRP publication 66 : Human Respiratory Tract Model for Radiological Protection in 1994 (ICRP 94) [5]. This model will be presented in this report with several additional details. The model was used to calculate the dose conversion coefficient DCC, to estimate the radiation doses and risks from inhaled radionuclides and to asses the radioactivity inhaled from measurements of activity in the body excretion. The Commission assigned numerical values of many model parameters known as "default" or "reference" values representing typical values. However there are situations where it is necessary to obtain more realistic dose estimations by using parameters that are more specific to given circumstances.

Recently, a new ICRP publication (ICRP Vol. 32) [6] has been issued to give guidance on applying the Human Respiratory Track Model in some specific situations. Applications of default and specific values of parameters involved in respiratory tract modeling was discussed in this newest issue of ICRP which was devoted to lung modeling.

\section{Human Respiratory Tract Model (HRTM)}

HRTM was described in a very comprehensive report [5] and here some the most 
important characteristics will be summarized.

Requests in HRTM :

-To apply on males and females of all ages including children.

-To apply to a whole range of aerosols particles, gases and vapors.

-To provide good estimates of doses and to be suitable for interpreting the monitoring data.

-To use material specific information, particularly those related to absorption from lung to the blood.

-To calculate dose in all parts of the respiratory tract separately and effective dose in the whole lung.

HRTM consists of several sub-models as follows :

Morphometric model describes shapes and sizes of organs and tissues in the respiratory tract.

Physiological model gives breathing parameters which depend on the body size, level of physical activity and age.

Radiobiology, describes sensitive cells that are targets for alpha radiation emitted by radon progeny.

Deposition model calculates the fraction of inhaled particles deposited in each organ of the respiratory tract.

Clearance model describes the clearance of deposited activity to other organs or out of the body through different clearance mechanisms.

Dosimetric model calculates doses in regions of respiratory tract by using the equilibrium activity and the absorbed fraction of alpha particles.

\subsection{Morphometric model :}

This model was described in Chapter 2 and Annex A of ICRP Publication 66 (ICRP1994) [5]. The respiratory tract consists of two parts, Extrathoracic airways (ET) and Thoracic airways $(\mathrm{TH})$.

$E T$ is divided into $E T_{1}$, anterior nasal passage, and $E T_{2}$, the posterior nasal passage, oral passage, pharynx, larynx and lymphatic tissue $\left(\mathrm{LN}_{\mathrm{ET}}\right)$. $\mathrm{TH}$ is divided into the bronchial region ( $\mathrm{BB}$, generations $0-8$, trachea is generation 0 ), bronchiolar (bb, generations 9-15) and alveolar - interstitial (Al, generations $\geq 16$, gas exchange region and lymphatic tissue $\mathrm{LN}_{\mathrm{TH}}$ ). All these regions are presented in Figure 1.

Dimensions of airways and other relevant information (mass, volume, surface area etc.) are given for reference workers and scaled to other members of population : 3- month old infants, 1-, 5-, 10- and 15-years old children and adults. Scaling factors according to the subject height are given in ICRP Publication 66 [5]. It is not 


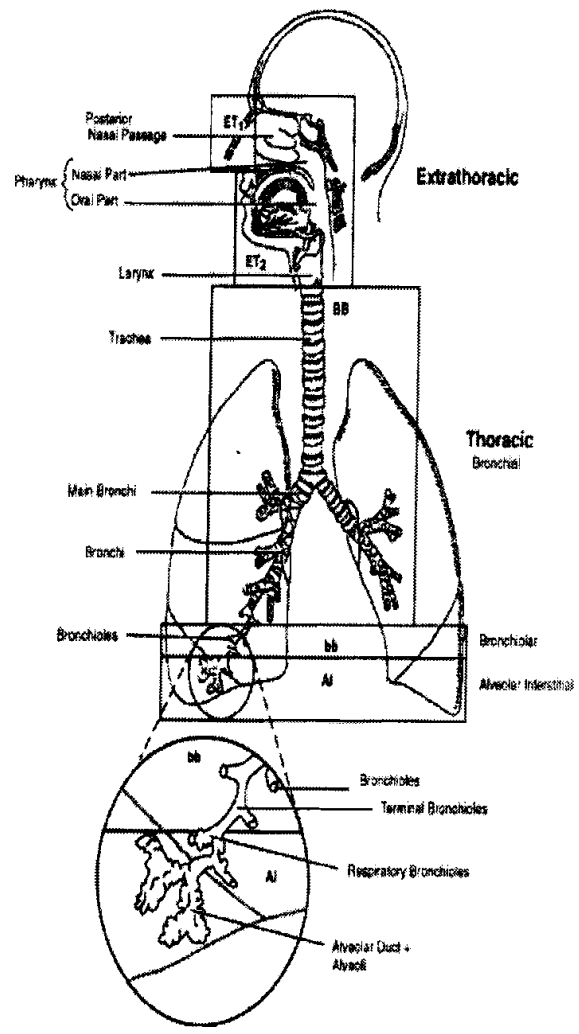

Figure 1. Morphometric model of respiratory track according to ICRP 66 [Ref : ICRP 66].

expectable that application of specific values would give some large difference in comparison when calculation is done for reference values, because there is compensating effect when the body increases : bigger body intakes more activity due to the larger air volume inhaled - this will increase the dose; on the other side, the mass of sensitive tissue is also larger - this decreases the dose.

\subsection{Physiology :}

Deals with air flow in to and out of respiratory tract. Several typical variables are used here :

Ventilation rate, $V$ is volume of air inhaled per unit time $\left(\mathrm{m}^{3} / \mathrm{h}\right)$.

Tidal volume, $V_{T}$, is volume of air inhaled per one breath $\left(\mathrm{L}^{-1}\right)$.

Breathing frequency, $f_{R}$, is number of breath per minute $\left(\mathrm{min}^{-1}\right)$.

Breathing mode is distribution of inhaled air between nose and mouth.

Total lung capacity, TLC is volume of air in the lung at maximum inhalation.

Functional residual capacity, FRC, is the volume of air left in the lung after normal exhalation. 
Ventilation rate obtained from tidal volume and breathing frequency is used to derive intake if exposure conditions are known.

Reference respiratory values for a general Caucasian population are given in [5], in Table 7 of Publication 66 for 6 age group : 3 months infants, 1 -, 5- 10, 15 - male and female, and adult male and female. Table 8 of ICRP Publication 66 gives respiratory parameters for same population group and age groups at different levels of activity (sleep, sitting, light and heavy exercise). In addition Table 9 of ICRP Publication 66 gives examples of variations of respiratory values for different ethnic groups (Japan, China, India, US, Senegal and Zimbabwe).

It is assumed that the reference subject is nose breathers, i.e. total air pass through the nose. Mouth breathing is also considered in ICRP Publication 66

The choice of default is based on whether the subject is worker or member of the general public. Then the age of the subject is taken into account. Finally the level of physical activity has to be defined.

Respiratory parameters influence regional deposition and final dose. Deposition in $\mathrm{BB}$ region increases with increasing exercise. Breathing mode significantly influences the dose, because in the case on mouth breathing, there is less deposition in ET and larger in BB. It has been considered that deposition is not sensitive to the age. If the specific values are used for respiratory parameters, it is expected that dose would not change too much, except for breathing mode.

\subsection{Radiobiology :}

Probably the most important questions are : which cells are sensitive to alpha radiation and where are they located in bronchial epithelia. Radiobiology considers two type of cells, basal and secretory cells, as targets for alpha radiation. Target cells - sensitive tissues in different regions of HRT are :

$\mathrm{ET}_{1}$ and $\mathrm{ET}_{2}$ - basal cells;

BB - basal and secretory cells;

bb - secretory cells;

Al - target cells are distributed all over the region, and whole region is a target.

The cross sections through the airway wall is given in Figure 2 ( $a$ and $b$ ).

The ICRP66 dosimetric model [5] is based on the assumption of different sensitivities of various tissues and cells in the human respiratory tract to ionizing radiation, so that calculations of doses in different regions are necessary. All regions of the human respiratory tract are sensitive to radiation, but with different sensitivities. The cells and tissues at risk are identified and their relative radio-sensitivities are attributed through radiobiology. The report recommended factors for weighting the doses in different tissues, so that one value for the thoracic region and one value for the extrathoracic region can be obtained. 


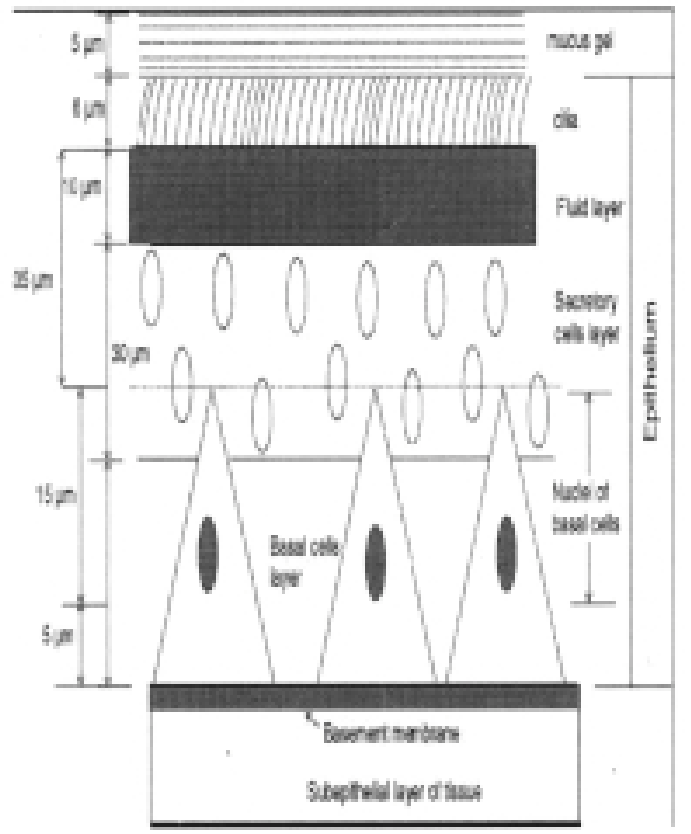

Figure 2a. Model of the bronchiolar wall in the BB region.

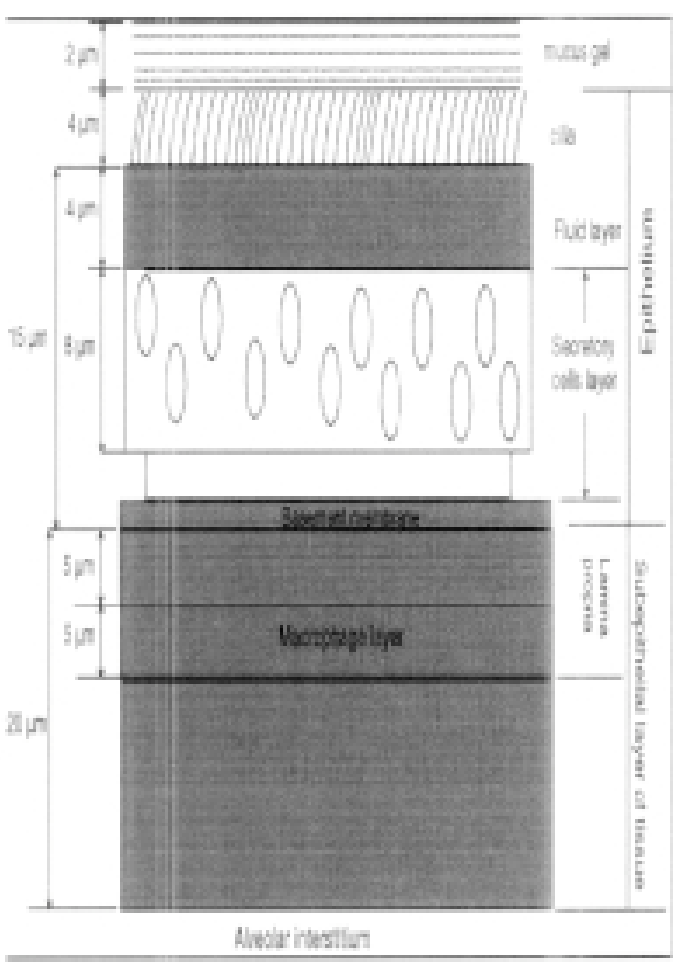

Figure 2b. Model of the bronchiolar wall in the bb region. 
It is not possible to determinate the locations of cancer origin; only the region of cancer (ET, BB, bb or Al) in the ICRP scheme can be determined. The existing set of experimental data is not enough for quantification of relative sensitivity of the BB, $\mathrm{bb}$, and $\mathrm{Al}$ regions. Therefore, the ICRP proposed equal risks for these three regions of the human lung. The equivalent doses should then be calculated according to the following equations :

$$
\begin{aligned}
& H_{\mathrm{ET}}=H_{\mathrm{ET} 1} A_{\mathrm{ET} 1}+H_{\mathrm{ET} 2} A_{\mathrm{ET} 2}+H_{\mathrm{LNET}} \\
& H_{\mathrm{TH}}=H_{\mathrm{BB}} A_{\mathrm{BB}}+H_{\mathrm{bb}} A_{\mathrm{bb}}+H_{\mathrm{Al}} A_{\mathrm{Al}}+H_{\mathrm{LNTH}} A_{\mathrm{LNTH}}
\end{aligned}
$$

where $H_{\mathrm{ET}}$ and $H_{\mathrm{TH}}$ are equivalent doses in the extrathoracic and thoracic regions, respectively, which are weighted according to the radiation detriment of the particular region, $H_{i}$ are the equivalent doses in the $i$-th region, and $A_{i}$ are factors for apportionment of radiation detriment for the $i$-th region, gien as : $A_{i}=0.333$ for $\mathrm{BB}$, bb; $\mathrm{Al}=0.001$ for $\mathrm{ET}_{1}$ and $\mathrm{LN}_{\mathrm{ET}}$ and finally $A_{i}=1$ for $\mathrm{ET}_{2}$.

The final step is calculation of the effective dose. The ICRP recommended the value 0.12 for the tissue weighting factor $w_{T}$ for the human lung. Other tissues of the respiratory tract were not listed in their report. In addition radiation weighting factor for alpha radiation is recommended 20; this value was criticized recently and there was a tendency to reduce it. In the newest ICRP proposal (now in draft, radiation weighting factor for alpha particles remains $w_{\alpha}=20$ ).

\section{Deposition}

Deposition of inhaled aerosols is one of the most important issues in the human respiratory tract model. It is considered as the main advance in comparison to the previous models. This problem has been extensively studied in the past and a comprehensive literature survey is given in Annex D of the ICRP report Publication 66 [5]. The deposition model is needed to provide the fraction of inhaled aerosols to be deposited in the human lung. The model described the deposition of aerosols in a very wide range of diameters, from atomic dimensions up to about $0.1 \mathrm{~mm}$. It is also applicable to different individuals under various exposure conditions, and enables calculations of deposition for gases and vapors.

The aerosols are mixed in air and they enter into the human lung during inhalation. A fraction of them is deposited in the lung while the rest is ejected from the body during exhalation. There are two groups of deposition processes, i.e., thermodynamic and aerodynamic deposition. The former is characteristic for smalldiameter particles and is often called diffusion or Brownian deposition. This type of deposition is caused by the random movement of the aerosols in the air stream. When the aerosols "touch" the wall of the airway tube, they can stay in that position. Aerodynamic deposition is more important for larger particles and there are two types of processes belonging to this group. The first one is "impaction" or inertial deposition. 
This deposition process takes place when the air stream changes the direction; some of the airborne aerosols with larger mass cannot adjust their directions of movement sufficiently quickly because of their inertia, and impact onto the wall of the airway tube. The second aerodynamic deposition process is gravitational sedimentation of aerosols. There are also other less important deposition processes like interception, which is important for fibers, etc. Deposition occurs in all compartments of the human lung, but with different efficiencies. The deposition pattern depends on the aerosol diameter as well as airflow characteristics. The behavior of small-size particles (which are deposited by diffusion) is described in terms of the thermodynamic diameter $d$-th and the diffusion coefficient $D$. Deposition of larger particles is described by the aerodynamic diameter $d_{\mathrm{ae}}$. The aerodynamic diameter is defined in terms of the equivalent particle volume diameter $d_{\mathrm{e}}$ (i.e., the diameter of a spherical particle with the same volume as the considered particle) by the following

$$
d_{\mathrm{ae}}=d_{\mathrm{e}} \sqrt{\frac{\rho C\left(d_{\mathrm{e}}\right)}{\chi \rho_{0} C\left(d_{\mathrm{ae}}\right)}}
$$

where $\rho$ is the particle density, $\rho_{0}=1 \mathrm{~g} \mathrm{~cm}^{-3}$ (unit density), $\chi$ the particle shape factor (its value is usually between 1 and 2), and $C$ is the so-called Cunningham correction slip factor given :

$$
C(d)=1+\frac{\lambda}{d_{\mathrm{e}}}\left(2.514+0.8 e^{-0.55 d_{\mathrm{e}} / \lambda}\right)
$$

where $\lambda=0.0712 \mu \mathrm{m}$ is the mean free path of air molecules at $37^{\circ} \mathrm{C}, 100 \%$ relative humidity and $760 \mathrm{mmHg}$ atmospheric pressure. The parameter $d_{\mathrm{ae}}$ appears on both sides of eq. (5) and iterations should be used in order to determine $d_{\mathrm{ae}}$.

The thermodynamic diameter is given in terms of its aerodynamic diameter by the following equation :

$$
d_{\mathrm{th}}=d_{\mathrm{e}} \sqrt{\frac{\chi \rho_{0} C\left(d_{\mathrm{ae}}\right)}{\rho C\left(d_{\mathrm{th}}\right)}}
$$

This equation, again, needs to be solved by iterations in order to find $d_{\mathrm{th}}$. If both kinds of deposition processes (thermodynamic and aerodynamic) are present, the combined effect is given as a quadratic sum :

$$
\eta=\sqrt{\eta_{\mathrm{th}}^{2}+\eta_{\mathrm{ae}}^{2}}
$$

where $\eta$ is the total deposited fraction, while $\eta_{\mathrm{th}}$ and $\eta_{\mathrm{ae}}$ are the thermodynamic and aerodynamic deposition fractions. For particles with thermodynamic diameters smaller than $0.1 \mu \mathrm{m}$, diffusion deposition is dominant. If the aerodynamic diameter is larger than $1 \mu \mathrm{m}$, aerodynamic deposition is more important. Instead the equivalent diameter, $d_{\mathrm{e}}$, the quantities AMAD and AMTD are frequently used. AMAD - activity median 
aerodynamic diameter, fifty percent of the activity in the aerosol is associated with particles of aerodynamic diameter greater than AMAD. AMTD - activity median thermodynamic diameter, fifty percent of the activity in the aerosol is associated with the particles of thermodynamic diameter larger than the AMTD.

The ICRP66 deposition model estimates regional deposition, i.e., deposition in each anatomical region of the respiratory tract. A semi-empirical approach has been used to describe the regional deposition. Relative simple algebraic equations derived from experiments and theory are used for the deposition model.

Each breath is represented by a tidal flow of air that carries particles through each anatomical region which is represented by one or more filters in series. A filter $j$ has two parameters, i.e., the volume $\nu_{j}$ and the filtration efficiency $\eta_{j}$, and $\Phi_{j}$ is the fraction of the tidal volume (denoted by $V_{t}$ ) which passes through the filter $j$. During inhalation, smaller and smaller fractions of the tidal volume pass through the filters in turn, which are determined by the cumulative volumes of the preceding filters. During exhalation, the same volume of air passes through the same filters as those during the inhalation. The filtration efficiency $\eta_{j}$ of the filter $j$ is the fraction of particles incident on the filter which is deposited.

The filtration efficiency (which is equal to the deposition efficiency) for an anatomical region is given in the form

$$
\eta=1-e^{a R^{p}}
$$

where $a$ and $p$ are parameters and $R$ is a function of the particle diameter and flow rate. The function in previous equation is given separately for thermodynamic and aerodynamic depositions, as well as for nasal and mouth breathing.

Aerosols consist of particles with different size, which are usually distributed according to the log-normal distribution. The parameters characterizing the deposition of activity in the respiratory tract are the activity median aerodynamic diameter (AMAD) for aerodynamic deposition, and the activity median thermodynamic diameter (AMTD) for thermodynamic deposition. The total deposition of polydispersed aerosols in an anatomical region is obtained as a weighted sum of the deposition of monodispersed aerosols. The weighting is carried out according to the fraction of aerosols within the specific diameter range.

ICRP Publication 66 recommended a set of values for the different variables in the deposition model described above. The following default values were recommended: AMAD $=5 \mu \mathrm{m}$ and geometric standard deviation $\sigma_{g}=2.5$ for occupational exposure; AMAD $=1 \mu \mathrm{m}$ and $\sigma_{g}=2.47$ for indoor and outdoor exposure of the general population; $\rho=3 \mathrm{~g} \mathrm{~cm}^{-3}$ for the particle density; $\chi=1.5$ for the particle shape factor. Reference values of breathing parameters are given in ICRP for six reference subject 3-months old infant, 1-, 5-, 10-, and 15-years old children and adults. Regional deposition as a 
function of aerosols diameter is given in ICRP Publication 66 in the form of graphs and Tables.

\subsection{Clearance :}

The materials deposited in the human respiratory tract are cleared with different mechanisms. These processes transfer the radioactivity from the lung into other organs and tissues in the body, so consideration of the clearance is also important for dose determination for other organs of the body. Extensive studies of clearance of materials deposited in the lung have been performed in the past. The literature survey about clearance is given in Annex $\mathrm{E}$ of the ICRP Publication 66 [5]. However, many uncertainties involved in clearance still remain. The materials deposited in the human lung are cleared through three main routes:

(1) into blood by absorption;

(2) to the gastrointestinal tract;

(3) to regional lymph nodes via lymphatic tubes.

First route is considered as absorption, while 2 and 3 are translocation. The clearance rate $\lambda_{i}$ defined as the part of materials $\lambda_{i}=\left(d R_{i}(t) / d t\right) / R_{i}(t)$ cleared per unit time. The total clearance rate is the sum of the rates due to individual processes. Clearance of an anatomical region is represented by the three routes as shown in Figure 3.

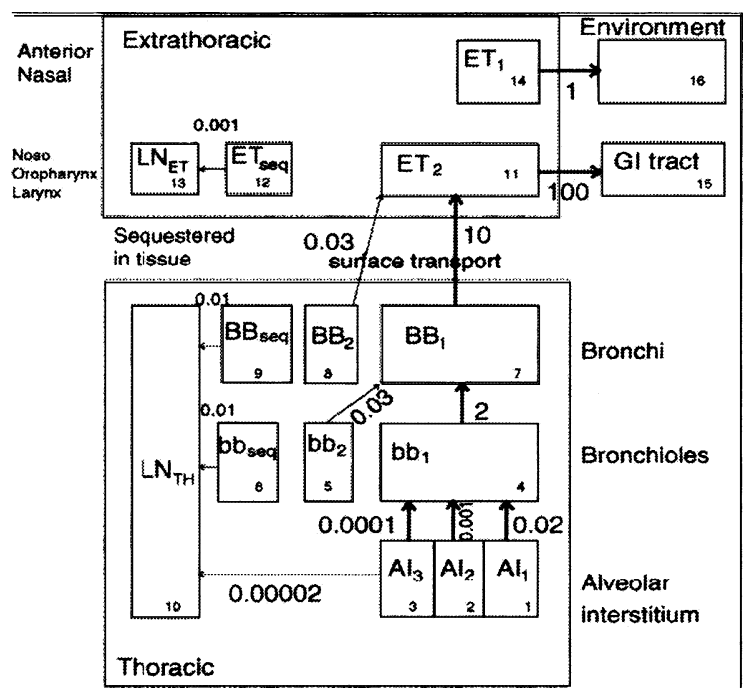

Figure 3. Compartment model of particle transport from each region. The transport rate is given in $\mathrm{d}^{-1}$.

The materials deposited in the $\mathrm{BB}$ and bb regions are subjects of fast clearing. These materials are dissolved in the mucous layer lining the surface of the airway tubes. The mucous moves upward and carries the dissolved particles towards the pharynx. In this way, the deposited particles are transferred from the site of deposition. 
The velocity of the mucous is a critical parameter. ICRP Publication 66 [5] recommended the values 10 and $2 \mathrm{~d}^{-1}$ for the mucous clearance rate from the $B B$ region to the pharynx and from the bb region to the $\mathrm{BB}$ region, respectively.

Part of the materials deposited in the BB and bb regions is cleared slowly. For this reason, slowly cleared compartments (numbers 5 and 8) are introduced. The particles are transported from these compartments through the airway surface. The clearance rate from this compartment is taken as $0.03 \mathrm{~d}^{-1}\left(t_{1 / 2}=23 \mathrm{~d}\right)$. Prolonged retention in the airway wall is described by the sequestration compartments $\mathrm{BB}_{\text {seq }}$ and $\mathrm{bb}_{\text {seq. }}$ ICRP66 assumes that a very small fraction as 0.007 of the deposited material is sequestered in the $\mathrm{BB}$ and bb regions. The sequestered activity is cleared to the lymph nodes with the rate of $0.01 \mathrm{~d}^{-1}$. The alveolar interstitial region is cleared slower than the BB or bb regions. Retention of insoluble particles in the alveolar region can be very long, even longer than a year. Experimental data suggested that the $\mathrm{Al}$ anatomical region should be considered as three sub-compartment regions, namely, $\mathrm{Al}_{1}$, $\mathrm{Al}_{2}$ and $\mathrm{Al}_{3}$, as shown in Figure 3. The clearance rates from these sub-compartments towards the bb region are $0.02,0.001$ and $0.0001 \mathrm{~d}^{-1}$.

The commission also assumes that absorption into blood happened with the same rate $s(t)$ in all considered regions except $\mathrm{ET}$, where there is no absorption. This process consists of two steps. The first one is dissolution of the material into a form that blood can uptake. The second one is the absorption of dissolute materials into blood. Absorption into blood is very dependent on the chemical form of the deposited material. The absorption rates for different materials have a very wide range from 100 $\mathrm{d}^{-1}$ (for very soluble materials) up to $0.0001 \mathrm{~d}^{-1}$.

In ICRP30 [4], the DWY classification scheme for the total particle clearance was presented. In ICRP Publication 66 [5], the new classification $F$ (fast), M (moderate), and $S$ (slow) was adopted, and referred only to absorption into blood. Type $\mathrm{F}$ denotes the particles which are absorbed fast from $\mathrm{BB}, \mathrm{bb}$ and $\mathrm{Al}$, and $50 \%$ of materials deposited in $\mathrm{ET}_{2}$. For type $\mathrm{M}, 70 \%$ of the materials deposited in $\mathrm{Al}$ reach the blood, while $10 \%$ of those deposited in the BB and bb regions and $5 \%$ of those in $\mathrm{ET}_{2}$ also reach the blood. Type $S$ denotes the materials that are little absorbed from $\mathrm{ET}, \mathrm{BB}$, bb into blood and $10 \%$ of those deposited in the Al region reach the blood. Absorption is a two stage process : dissociation of the particle in the form that can be absorbed into fluids, and absorption into fluid. Default parameters values are given in Table 1.

\subsection{Dose weighting scheme and calculation of effective dose :}

The dose $D_{\mathrm{BB}}$ in the $\mathrm{BB}$ region is obtained as the average of the dose $D_{\mathrm{BB} \text {,bas }}$ for basal cells and $D_{\mathrm{BB}, \mathrm{sec}}$ for secretory cells, i.e.,

$$
D_{\mathrm{BB}}=0.5\left(D_{\mathrm{BB}, \mathrm{bas}}+D_{\mathrm{BB}, \mathrm{sec}}\right)
$$


Table 1. Default parameters for clearance.

\begin{tabular}{lcccc}
\hline Type & \multicolumn{2}{c}{$\mathrm{F}$ (fast) } & \multicolumn{2}{c}{ Moderate $(\mathrm{M})$} \\
\hline $\begin{array}{l}\text { Fraction } \\
\text { dissolved }\end{array}$ & $f_{r}$ & 1 & 0.1 & 0.001 \\
rapidly & & & & \\
\hline Dissolution rate & & & & \\
\hline Rapid $\left(\mathrm{d}^{-1}\right)$ & $s_{r}$ & 100 & 100 & 100 \\
Slow $\left(\mathrm{d}^{-1}\right)$ & $s_{s}$ & - & 0.005 & 0.0001 \\
\hline
\end{tabular}

The dose $D_{\mathrm{bb}}$ in the bb region is equal to the dose for the secretory cells in this region, i.e.,

$$
D_{\mathrm{bb}}=D_{\mathrm{bb}, \mathrm{sec}}
$$

The mean dose $D$ for the respiratory track is then found as

$$
D=0.333\left(D_{\mathrm{BB}}+D_{\mathrm{bb}}+D_{\mathrm{Al}}\right)
$$

where $D_{\mathrm{Al}}$ is the absorbed dose in the Al region. There are two intrinsic assumptions in this weighting scheme. The first one is the equal sensitivity of basal and secretory cells in the BB region, which might not be true. The second one is the equal sharing of radiation detriment among the three regions ( $\mathrm{BB}, \mathrm{bb}$ and $\mathrm{Al}$ ), where the same weighting factor $A_{\mathrm{BB}}=A_{\mathrm{bb}}=A_{\mathrm{Al}}=0.333$ was applied to each of them. The remaining 0.001 was used for lymphatic tissues. This assumption is more critical because it is known that the majority of lung cancers have origins in the upper part of the T-B tree.

Dose equivalent, $H_{\mathrm{L}}$, is calculated by multiplying of average absorbed dose in respiratory tract $D$, with radiation weighting factor for alpha radiation $W_{\text {alpha }}=20$. The next step is multiplication of dose equivalent $H_{L}$ with tissue weighting factor $w_{L}=0.12$ to obtain effective dose, that is final results of dosimetric model.

\section{Program description}

Software that implemented the ICRP model Publication 66 [5] were available, namely, the LUDEP program and its smaller version RADEP for radon and its progeny. In addition to these, there is our home written program that follows ICRP66 recommendations as much as possible. These software enabled calculations of DCCs and analyses of influence of different relevant parameters on the lung dose.

Our software consists of several sets of programs. The first set calculates absorbed fraction AF, namely the fraction of alpha energy absorbed in sensitive tissues. Here, the source and the target should be defined. Although the absorbed fractions have already been given in Annex $\mathrm{H}$ in ICRP66 publication, we have prepared our own programs to calculated AFs. The same geometry was implemented as described in ICRP66 - infinite long straight cylinder with a layer of tissues. The reason 
for developing of this program was to enable calculation for different thickness of layers tissues. Results are given in [7]. Two programs were developed here; one is based on analytical approach given in [8] and [9]. Second program was written based on Monte Carlo Method and similar results were obtained by these two different approaches. Monte Carlo program also enables calculation of micro dosimetric quantities, specific energy and lineal energy [9-11]. It also enables calculation of absorbed fraction in bifurcation geometry that is more characteristic for human lung than long straight cylinders [12].

Absorbed fraction of alpha particles for different sources and targets are used as input parameters in the second set of programs that calculates dose conversion coefficients DCCs. To do such calculation one should define the values of various parameters that are divided in "subject related" parameters and "aerosol related" parameter. In addition, the exposure situation should be define, i.e. the concentration of radon and its short lived progeny expressed through the value of PAEC. Second set is consists of three programs, that may be run separately, and also altogether in sequence.

The first program, called DEPOSITION.F90 calculates deposition of monodisperse aerosols in human respiratory tract. Calculations is done for aerosols with diameters starting from $0.001 \mu \mathrm{m}$ and finished with a diameter of $5 \mu$. The step is variable, it is only $0.001 \mu \mathrm{m}$ for smaller aerosols in the region of unattached fraction and larger as $0.01 \mu \mathrm{m}$ or $0.1 \mu \mathrm{m}$ in the micrometer region. Program fully follows ICRP66 deposition model. The output is a file REG_DEP.DAT where the deposited fraction of monodsperse aerosols is given for all regions of HRTM.

Second program has two versions. The first one LOGSUMING.F90 perform summation according to log normal distribution assuming that both fraction, unattached and attached are mono modally distributed. Here parameters of aerosols like AMAD, AMTD, and geometric standard deviation, $\sigma_{g}$, are used. Second version of the program LOG_SUMING_3M.F90 performs summation assuming that attached fraction is three modal; here the AMAD, AMTD and $\sigma_{g}$ are used for all three modes. In addition the fraction of activity in different modes $f_{i}$ ( $i=$ nucleation, accumulation or coarse) is also needed for calculation. The results of LOG_SUMING.F90 are written in a file DEP_ORGANS.F90 where the deposited activity in various regions of aerosols distributed according to a given log normal distributions are given.

The third program CLEARANCE.F90 calculates clearance and transfer of deposited material in HRTM. Clearance model is a set of linear equation whose solution represents equilibrium activities in different regions of HRTM. From equilibrium activity we can calculate the number of alpha particles emitted per unit of time in different part of HRTM. By multiplying of these numbers with absorbed fraction the energy of alphas absorbed in target tissue is obtained. The ratio of absorbed energy and the mass of. 
tissue gives absorbed dose in that tissue. Program CLEARANCE.F90 made all these calculations and it also performs dose weighting, transforming absorbed dose in effective dose given in mSv. As a final step, effective dose is divided with assumed exposure (PAEC multiplied with the exposure time) to obtain DCC in mSv/WLM.

\subsection{Some results :}

Several calculations are presented here :

(a) Dependence of DCC on the transfer to blood rate $\lambda_{B}$ :

Transfer rate of deposited activity from lung to blood is important parameter in dose calculation. It depends dominantly on chemical form of inhaled aerosols. In some publication it was used $10 \mathrm{~h}$ as reference value, but this result was not fully verified experimentally. Dependence of DCC on $\lambda_{B}$ is presented in Figure 4. Strong increasing of DCCs for small $\lambda_{B}$ and saturation for larger is obvious. All other parameters were kept on their best estimation.

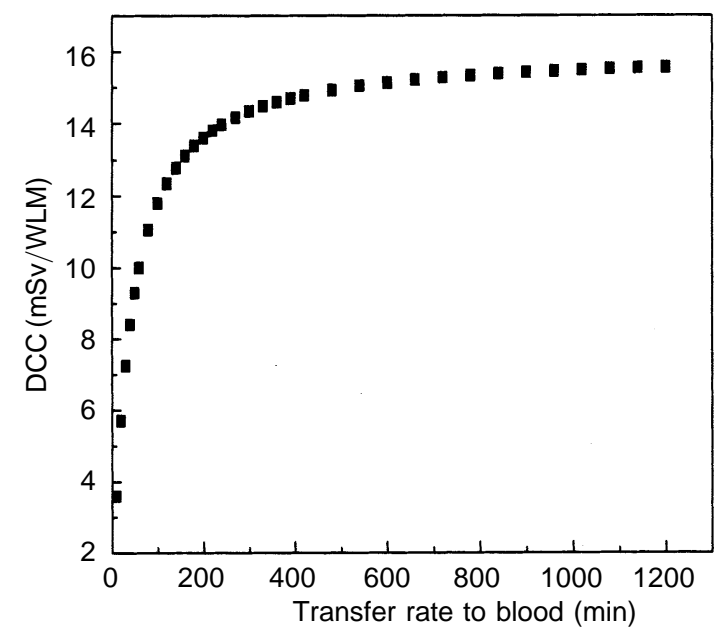

Figure 4. Dependence of DCC on transfer rate to blood.

(b) Dependence of DCC on breathing frequency $B$ :

Similarly to the previous, all parameters were kept on the best estimation, and dependence on breathing frequency is presented in Figure 5. Linear trend is seen in Figure 5.

(c) Dependence of DCC on unattached fraction $f$ :

Unattached fraction of radon progeny deposits in the upper part of HRT (BB) with very high efficiency. It is why the dose is strongly influenced by unattached fraction. Other parameters were also kept on the best estimation, and $f$ was varied $6 \%$ and $22 \%$. DCC increases from 12 to about $17 \mathrm{mSv} /$ WLM (Figure 6). 


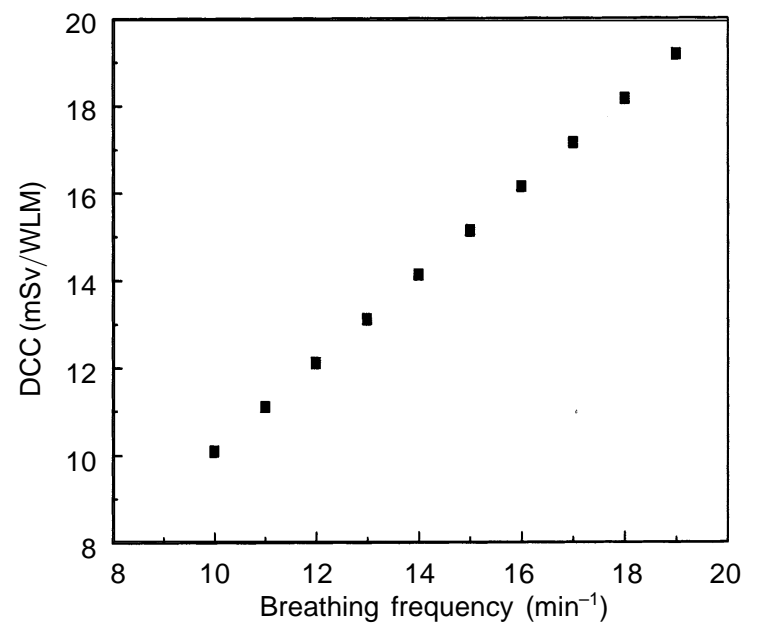

Figure 5. Dependence of DCC on breathing frequency.

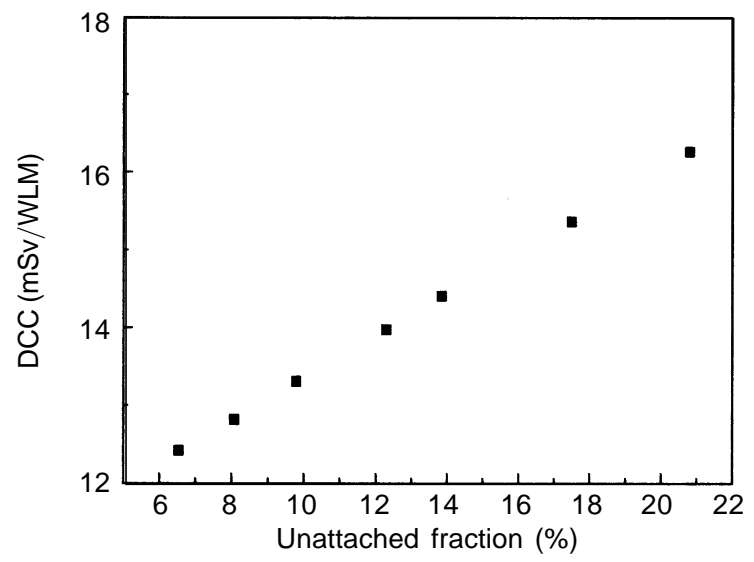

Figure 6. Dependence of DCC on unattached fraction.

\section{Conclusion}

Home made computer program for dose calculations is presented in this talk. As an advantage of such work we emphasize that many mid-results become available and could be used for other calculations and investigation.

The main result of HRT dosimetric model is dose conversion coefficient, i.e. effective dose per unit exposure (mSv/WLM). A lot of effort has gone in modeling and computing. However, the result is more/less disappointing. Namely, DCC obtained from dosimetric model is about (14-15) $\mathrm{mSv} / \mathrm{WLM}$ for home irradiation. Similar results are found by another computer programs.

DCC can be determined from epidemiologic studies. DCC obtained in this way is about $4 \mathrm{mSv} / \mathrm{WLM}$. This significant discrepancy has not been fully resolved yet. 


\section{References}

[1] UNSCEAR United Nation Scientific Committee on the Effects of Atomic Radiation. Sources, effects and risk of ionizing radiation (New York : United Nations) (1988)

[2] International Commission on Radiological Protection, Recommendation of International Commission on Radiological Protection, Report of Committee II on Permissible Dose for Internal Radiation (1959). ICRP Publications 2 (Oxford: Pergamon Press) (1960)

[3] Task Group on Lung Dynamics (TLGD), Health Phys. 12173 (1966)

[4] International Commission on Radiological Protection, Limits for Intakes of Radionuclides by Workers, ICRP Publication 30 (Oxford : Pergamon Press) (1979)

[5] International Commission on Radiological Protection, Human respiratory tract model for radiological protection : A report of a Task Group of the International Commission on Radiological Protection, ICRP Publication 66 (Oxford : Pergamon Press) (1994)

[6] International Commission on Radiological Protection, Guied for Practical Application of the ICRP Human Respiratory Tract Modal. Annal of the ICRP Vol 32 (1-2) (2002)

[7] D Nikezic, K N Yu and D Vucic Physics in Medicine and Biology 461963 (2001)

[8] A K M M Haque British Journal of Applied Phys. 18657 (1967)

[9] D Nikezic and K N Yu Radiation and Environmental Biophysics 40207 (2001)

[10] D Nikezic and K N Yu Radiation Research 15792 (2002)

[11] D. Nikezic and K N Yu International J. Radiation Biology. 78(7) 605 (2002)

[12] D Nikezic, K N Yu Radiation Environment Biophysics 4249 (2003) 